\title{
Dynamic Flow Impacts Cell-Particle Interactions: Sedimentation and Particle Shape Effects
}

Mattias Björnmalm, Matthew Faria, Xi Chen, Jiwei Cui, and Frank Caruso*

ARC Centre of Excellence in Convergent Bio-Nano Science and Technology, and the

Department of Chemical and Biomolecular Engineering, The University of Melbourne, Parkville, Victoria 3010, Australia 
ABSTRACT: The interaction of engineered particles with biological systems determines their performance in biomedical applications. Although standard static cell cultures remain the norm for in vitro studies, modern models mimicking aspects of the dynamic in vivo environment have been developed. Herein, we investigate fundamental cell-particle interactions under dynamic flow conditions using a simple and self-contained device together with standard multiwell cell culture plates. We engineer two particle systems and evaluate their cell interactions under dynamic flow, and we compare the results to standard static cell cultures. We find substantial differences between static and dynamic flow conditions and attribute these to particle shape and sedimentation effects. These results demonstrate how standard static assays can be complemented by dynamic flow assays for a more comprehensive understanding of fundamental cell-particle interactions. 


\section{INTRODUCTION}

The human body is in constant, but well-controlled, flux. ${ }^{1}$ This includes the flow of a wide range of nutritional, waste, and signaling substances in blood, lymph, and interstitial fluid at flow rates that range from decimeters per second (large blood vessels) ${ }^{2,3}$ to micrometers per second (interstitial convection). ${ }^{4,5}$ The ability to recapitulate aspects of these dynamic biological environments in vitro is therefore of interest, both for probing the biology and for evaluating fundamental bio-nano interactions of nanoengineered particles aimed at biomedical applications. ${ }^{6-9}$

The application of particles in biomedicine is, in large part, governed by their bio-nano interactions, with both the properties of the particles and the biological environment being of primary importance. ${ }^{10-15}$ In vitro cell cultures have been, and continue to be, an integral part in research efforts aimed at elucidating fundamental bio-nano interactions. However, the static nature of conventional assays (e.g., standard multiwell plates) only captures part of the dynamic in vivo environment. Some commercial systems for flow-based cell cultures exist, although these are typically made for specific suspension cell types or for specific assays, such as leukocyte recruitment assays. ${ }^{16}$ Additionally, it has been demonstrated that the effects of diffusion and sedimentation in standard in vitro assays can cause large differences in the cellular dose of different particles. ${ }^{17-19}$

Microfluidic devices have proven to be a powerful tool to complement conventional assays. ${ }^{16,20,21}$ Examples include probing tissue transport, ${ }^{22,23}$ blood flow behavior, ${ }^{24}$ deformability, ${ }^{25-28}$ and shape-effects of engineered particles under flow. ${ }^{29-31}$ Microfluidic systems are highly suitable for well-controlled laminar flow environments, but these only reflect part of the in vivo milieu, and laminar flow can be challenging to combine with more 
dynamic/chaotic flow and mixing. ${ }^{32,33}$ Furthermore, widespread adoption of microfluidic devices remains limited, partly as the skills and expertise required for designing and developing these devices is different to those required for standard cell cultures. ${ }^{16}$

Herein, we present a simple, self-contained, and programmable mixing device (compatible with standard multiwell plates) for investigating fundamental cell-particle interactions under dynamic flow conditions (Figure 1). We engineer two particle systems and investigate the effects of particle shape and sedimentation on cell-particle association under dynamic flow conditions. Substantial differences in how particles interact with cells under the dynamic flow conditions - compared to standard static cell cultures-are found and possible explanations are discussed.

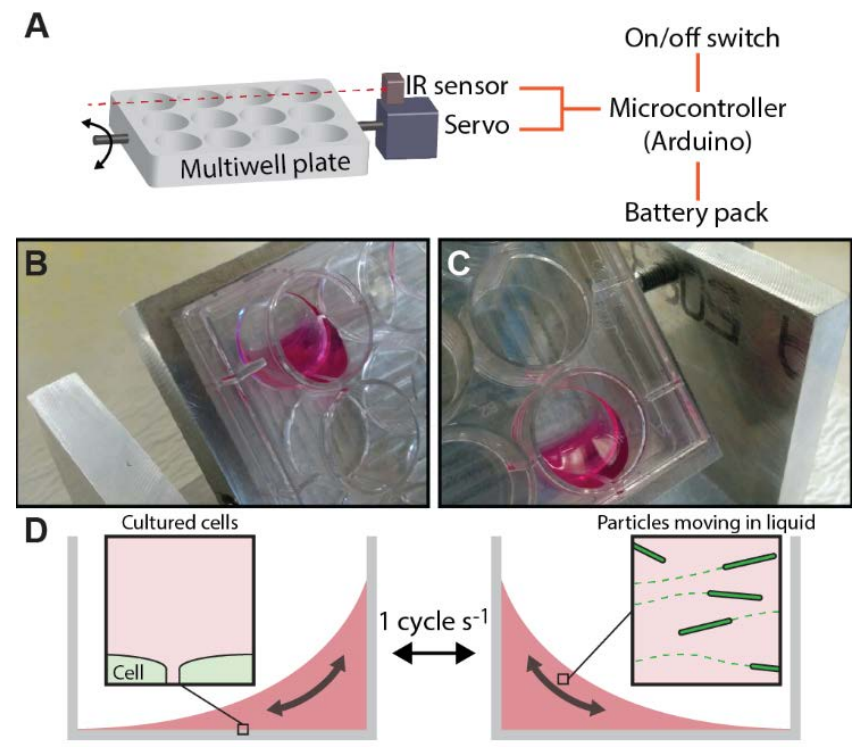

Figure 1. Simple, self-contained mixing device for investigating fundamental cell-particle interactions under dynamic flow conditions. A) A microcontroller (“Arduino”, an open-source electronics platform) is programmed with instructions and inserted into a circuit with an on/off switch, an infrared (IR) sensor and a servo connected to a multiwell plate holder. B,C) Photos of wells in a 12-well plate in starting position (B) and half-way through one cycle (C). D) Scheme 
illustrating the movement of the liquid in relation to the adherent cells and the movement of the rod-shaped particles induced by the moving liquid for typical dynamic flow conditions (1 cycle per second).

\section{EXPERIMENTAL SECTION}

Materials. Dulbecco's phosphate-buffered saline (DPBS), hydrofluoric acid (HF, 48 wt\%), 1-(3-dimethylaminopropyl)-3-ethylcarbodiimide (EDC), ammonium fluoride $\left(\mathrm{NH}_{4} \mathrm{~F}\right), 4$ (4,6-dimethoxy-1,3,5-triazin-2-yl)-4-methylmorpholinium chloride (DMTMM), dithiothreitol (DTT), poly(N-vinylpyrrolidone) (PVPON), thiazolyl blue tetrazolium bromide (MTT), penicillin-streptomycin, and ammonium hydroxide solution (28-30\%) were obtained from Sigma-Aldrich (Australia). Poly(methacrylic acid, sodium salt) (PMA, $M_{\mathrm{w}} 15 \mathrm{kDa}, 30 \mathrm{wt} \%$ solution in water) was from Polysciences, Inc. (USA). Alexa Fluor 488 (AF488) Cadaverine, AF488 wheat germ agglutinin (WGA), Alexa Fluor 633 (AF633) hydrazide, Hoechst 33342, LIVE/DEAD Viability/Cytotoxicity Kit, fetal bovine serum (FBS), and Dulbecco's Modified Eagle Medium (DMEM) with GlutaMAX Supplement were purchased from Life Technologies (Australia). MycoAlert Mycoplasma Detection Kit was purchased from Lonza (Australia). Thiolfunctionalized PMA $\left(\mathrm{PMA}_{\mathrm{SH}}\right)$ was prepared as described previously. ${ }^{34}$ Electronic components, including IR sensor, servo and wires were obtained from a local electronics retailer (Jaycar Electronics, Australia). Rechargeable battery packs (5V; 16,800 mAh capacity) were obtained from Gorilla gadgets (USA). The water used in all experiments was prepared in a three-stage Millipore Milli-Q Plus 185 purification system and had a resistivity greater than $18.2 \mathrm{M} \Omega \mathrm{cm}$.

Synthesis of hybrid core-shell PMA particles and capsules. The details of particle

synthesis and characterization have been described previously. ${ }^{18}$ Briefly, solid core/mesoporous shell (SC/MS) silica templates were prepared and infiltrated with polymer after pore 
enlargement. To facilitate loading of negatively charged PMA into the porous shells of the SC/MS templates, 3-aminopropyltriethoxysilane was used to introduce primary amine groups through a postgrafting method. After polymer infiltration into the porous shell of the templates the PMA was cross-linked using a thiol-disulfide exchange strategy. Well-dispersed PMA capsules with negligible aggregation were obtained by dissolving the SC/MS silica template in 2 M HF / $8 \mathrm{M} \mathrm{NH}_{4} \mathrm{~F}$ solution ( $\left.\mathrm{pH} \sim 5\right)$. Caution! HF is dangerous. Care should be taken when handling HF solution and only small quantities should be prepared. The core-shell particles and capsules were labelled with Alexa Fluor 488 Cadaverine using EDC-based chemistry.

Synthesis of spherical and rod-shaped PMA SH $_{\mathrm{SH}}$ capsules. The details of particle synthesis and characterization have been described previously. ${ }^{34}$ Briefly, silica templates were coated with PMA $\mathrm{SH}_{\mathrm{SH}} / \mathrm{PVPON}$ through layer-by-layer assembly ${ }^{35}$ under acidic conditions (pH 4), followed by crosslinking of $\mathrm{PMA}_{\mathrm{SH}}$ thiol groups through oxidation. Well-dispersed $\mathrm{PMA}_{\mathrm{SH}}$ capsules with negligible aggregation were obtained after dissolution of the silica templates in hydrofluoric acid and removal of the sacrificial PVPON layers by washing at $\mathrm{pH}$ 7.4. The capsules were fluorescently labeled by incubation with Alexa Fluor 633 hydrazide and DMTMM.

Characterization. For hybrid core-shell PMA particles and capsules, transmission electron microscopy (TEM) and cryo-electron tomography was used to examine the particle morphologies, dimensions, and densities, as described previously. ${ }^{18}$ The TEM samples $(1 \mu \mathrm{L})$ in water were placed onto strong carbon film-coated copper grids (ProSciTech, Australia) and allowed to air-dry before TEM analysis (FEI Tecnai G2 Spirit, operated at $120 \mathrm{kV}$ ). For cryoelectron microscopy, sample vitrification was performed using an automated plunge freezing robot (Vitrobot). For imaging and tomography, the samples were studied using a FEI Tecnai G2 
Spirit TEM operated at $120 \mathrm{kV}$ and processed as described previously. ${ }^{18}$ For spherical and rodshaped PMA $\mathrm{SH}_{\mathrm{SH}}$ capsules dimensions were determined using TEM analysis (FEI Tecnai G2 Spirit, operated at $120 \mathrm{kV}$ ), as described previously. ${ }^{34}$ Particle counting and cellular association studies were performed using an Apogee flow cytometer (Apogee A50 Micro). Fluorescence microscopy images of particles and capsules were obtained using an Olympus IX71 inverted fluorescence microscope. Results of super-resolution fluorescence microscopy and atomic force microscopy analysis have previously been reported for the particle systems used in this study. ${ }^{18,34}$

Dynamic flow device. See Figures S1-S3 in the Supporting Information for photographs of the device. The multiwell plate holder was machined out of a $14 \mathrm{~cm} \times 9.5 \mathrm{~cm} \times 1 \mathrm{~cm}$ metal plate using a milling machine. The support structure was also assembled from metal plates, one of them machined to hold the servo (Figures S2 and S3). The Arduino was programmed (see the Supporting Information) with the software and instructions provided with the Arduino. An IR sensor was used to provide the Arduino with feedback on the position of the multiwell plate holder to keep the tilting stable over time, e.g., adjusting for any drift in the servo. The Arduino, battery pack, IR sensor, and servo were connected using standard electrical wire and a solderless breadboard. A mixing frequency of 1 second per cycle was chosen for the proof-of-concept study reported herein. During each cycle, the liquid in each well moved from side to side (Figure 1BD). For the 12-well plates that were used herein (where each well has a diameter for around 2 $\mathrm{cm})$ this corresponds to an average flow rate of $\sim 2 \mathrm{~cm} \mathrm{~s}^{-1}$. This flow rate is within the range of physiological flow rates present in the human body, which ranges from decimeters per second (large blood vessels) ${ }^{2,3}$ to micrometers per second (interstitial convection). ${ }^{4,5}$ While this proof-ofconcept study focuses on one average flow rate (to facilitate comparison of multiple particle 
systems under dynamic mixing), other average flow rates can be obtained by tuning the servo (the tilting frequency) and by using well plates with other geometries and dimensions.

Cell culture. NIH/3T3 cells were kept in culture (DMEM with 10\% FBS) in a standard humidified incubator $\left(37^{\circ} \mathrm{C}, 5 \% \mathrm{CO}_{2}\right.$ ) and passaged before confluence. Cells were cultured without antibiotics and with no sign of bacterial infection. The cell cultures also tested negative for mycoplasma infection (MycoAlert kit).

Viability testing. 80,000 cells in 1 mL DMEM with $10 \%$ FBS were added into each well of cell culture-grade 12 -well plates and allowed to adhere overnight $\left(37^{\circ} \mathrm{C}, 5 \% \mathrm{CO}_{2}\right)$ in standard static set-up (multiwell plate just in incubator without mixing). After overnight incubation, the multiwell plate was place in the multiwell plate holder of the mixing set-up (Figure S1) and the mixing was started. Tilting had been pre-programmed to achieve liquid movement seen in Figure 1B,C (close to maximum tilting without any liquid spilling out of well) and each cycle lasted $~ 1$ s. The whole self-contained mixing set-up was kept on top of a plastic document holder (A4 size) on a shelf inside the humidified incubator $\left(37^{\circ} \mathrm{C}, 5 \% \mathrm{CO}_{2}\right)$. After $24 \mathrm{~h}$ incubation under dynamic flow conditions the mixing was stopped and the cells were stained using a LIVE/DEAD staining kit and evaluated using fluorescence microscopy. The experiment was also repeated with the LIVE/DEAD kit replaced by an MTT assay with readout performed using an Infinite M200 microplate reader (Tecan, Switzerland). In both experiments a static control multiwell plate was included in which the cells were treated the same way as for the mixing set-up but kept static instead of under dynamic flow during incubation.

Cell-particle experiments. 80,000 cells in 1 mL DMEM with 10\% FBS were added into each well in a cell culture-grade 12 -well plates and allowed to adhere overnight $\left(37^{\circ} \mathrm{C}, 5 \% \mathrm{CO}_{2}\right)$ in standard static set-up (multiwell plate just standing in incubator without mixing). After 
overnight incubation, the media was replaced with $1 \mathrm{~mL}$ particle solution for each well and the multiwell plate was then placed in the multiwell plate holder of the mixing set-up (Figure S1) and the mixing was started. For PMA hybrid core-shell particles and capsules a cell to particle ratio of 1 to 5000 was used. ${ }^{18}$ For $\mathrm{PMA}_{\mathrm{SH}}$ spherical and rod-shaped capsules a cell to particle ratio of 1 to 100 was used. ${ }^{34}$ The particle solutions consisted of particles/capsules (at cell-toparticle ratios listed above) added into DMEM supplemented with penicillin, streptomycin and 10\% FBS. Tilting had been pre-programmed to achieve liquid movement seen in Figure 1B,C (close to maximum tilting without any liquid spilling out of well) and each cycle lasted $\sim 1 \mathrm{~s}$. The whole self-contained mixing set-up was kept on top of a plastic document holder (A4 size) on a shelf inside the humidified incubator $\left(37^{\circ} \mathrm{C}, 5 \% \mathrm{CO}_{2}\right)$. After $24 \mathrm{~h}$ incubation the cells were washed three times with DPBS and harvested by trypsinization. Following centrifugation (300 g, $5 \mathrm{~min}$ ) the cell pellet was resuspended in $300 \mu \mathrm{L}$ DPBS and analyzed by flow cytometry. At least 10,000 cells were interrogated for each sample and experiments were performed at least in duplicate. For PMA capsules and core-shell particles the median and median absolute deviation of the AF488 signal (from associated particles) for the cell population was recorded (Figure S4). The fluorescence intensity was normalized between the two particle types by measuring the fluorescence intensity of the particles without cells (e.g., the fluorescence intensity of core-shell particles were $1.2 \times$ the fluorescence intensity of the polymer capsules). ${ }^{18}$ For the $\mathrm{PMA}_{\mathrm{SH}}$ spherical and rod-shaped capsules the percentage of cells positive for particles (assessed using AF633 signal from particles) was recorded (Figure S5), as done previously. ${ }^{34}$

\section{RESULTS AND DISCUSSION}

The mixing device was made using a holder for a multiwell plate attached to a servo controlled by a microcontroller (Figures S1-S3 in the Supporting Information). The whole self- 
contained device fits inside a plastic document holder (A4 paper size). When investigating cell viability, no substantial difference was observed between dynamic and standard static cell cultures (Figure 2). All particle types remained intact (with no visible damage) after exposure to dynamic cell culture conditions.

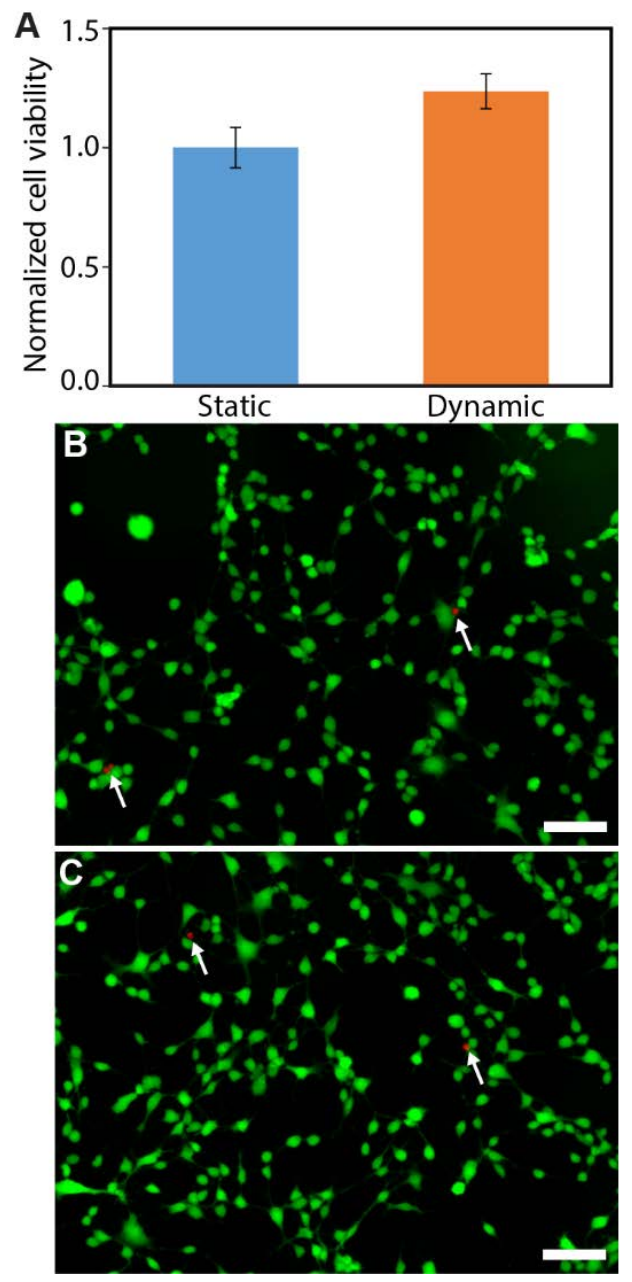

Figure 2. Viability of NIH/3T3 cells after 24 h under static and dynamic conditions. A) Cell viability measured using MTT assay. The slight difference observed is not statistically significant $(\mathrm{P}>0.05)$. B) Cell viability and morphology observed using a LIVE/DEAD staining kit after static cell culture. Live cells are green (calcein-AM) and dead cells are red (ethidium 
homodimer-1). Arrows indicate dead cells. C) Same as B) but after dynamic cell culture. Scale bars are $100 \mu \mathrm{m}$.

The effect of sedimentation and diffusion can be a significant factor when comparing cell-particle interactions using standard static assays. ${ }^{17,18}$ We engineered core-shell particles and polymer capsules based on mesoporous silica templating ${ }^{18}$ to investigate these effects under dynamic flow conditions (Figure 3). SC/MS silica templates (250 nm in diameter) were loaded with PMA and after cross-linking the template was dissolved. The particles were made in a single batch with the primary difference being that the silica template remains for one (core-shell particle) and has been dissolved for the other (polymer capsule). Therefore, a main difference between these particles is their effective densities (Table 1), and hence their sedimentation behavior.

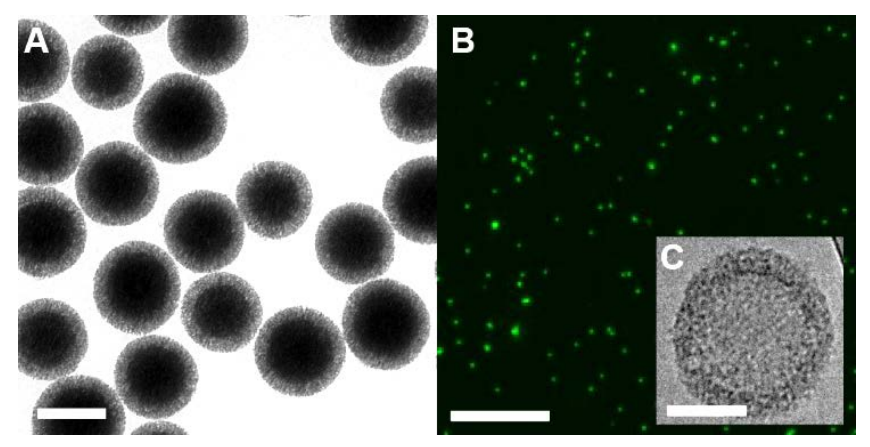

Figure 3. Templates and resulting PMA capsules after polymer infiltration, cross-linking and template dissolution. A) TEM image of the SC/MS silica templates. B) Fluorescence microscopy image of fluorescently labeled capsules. C) Cryo-TEM image of a PMA capsule. Scale bars are $200 \mathrm{~nm}(\mathrm{~A}), 5 \mu \mathrm{m}(\mathrm{B})$ and $100 \mathrm{~nm}(\mathrm{C})$.

Table 1. Size, shell thickness, and average density of hybrid core-shell PMA particles and capsules 


$\begin{array}{lccc}\text { sample } & \begin{array}{c}\text { diameter } \\ (\mathrm{nm})\end{array} & \begin{array}{c}\text { shell thickness } \\ (\mathrm{nm})\end{array} & \begin{array}{c}\text { average density } \\ \left(\mathrm{g} \mathrm{cm}^{-3}\right)\end{array} \\ \begin{array}{l}\text { core-shell } \\ \text { particles }\end{array} & 250 \pm 10 & 38 \pm 3 & 1.65 \\ \text { capsules } & 200 \pm 10 & 30 \pm 2 & 1.06\end{array}$

We hypothesized that dynamic flow could be used to equalize the cellular dose of particles by maintaining a well-mixed solution and preventing particles from settling. To investigate this, the two particle types were incubated with cells both under standard static and dynamic flow conditions (Figure 4). We have previously investigated how these particles interact with cells in 3D printed cell culture systems, and we have observed that the capsules (density $\sim 1.06 \mathrm{~g} \mathrm{~cm}^{-3}$ ) had substantially less cellular association compared to core-shell particles (density $\sim 1.65 \mathrm{~g} \mathrm{~cm}^{-3}$ ) which was explained by differences in sedimentation behavior (with differences in stiffness having no discernable effect). ${ }^{18}$ This is also visible here (Figure 4A, solid lines). However, interestingly, for dynamic flow conditions these effects are not observed and the cellular association for particles and capsules is similar. This is in agreement with the hypothesis that dynamic flow conditions introduce mixing that reduces or eliminates the effects of sedimentation (Figure 4B). If the liquid in a well of a 12-well plate on average moves a full well diameter during one cycle (Figure 1B-D) then this corresponds to an average flow velocity of $\sim 20 \mathrm{~mm} \mathrm{~s}^{-1}$, which is several orders of magnitude higher than the sedimentation velocities for these particles $\left(<1 \mu \mathrm{m} \mathrm{s}{ }^{-1}\right){ }^{18}$ Further details (including computational modeling) on the sedimentation and diffusion of these particles have been reported previously. ${ }^{18}$ 

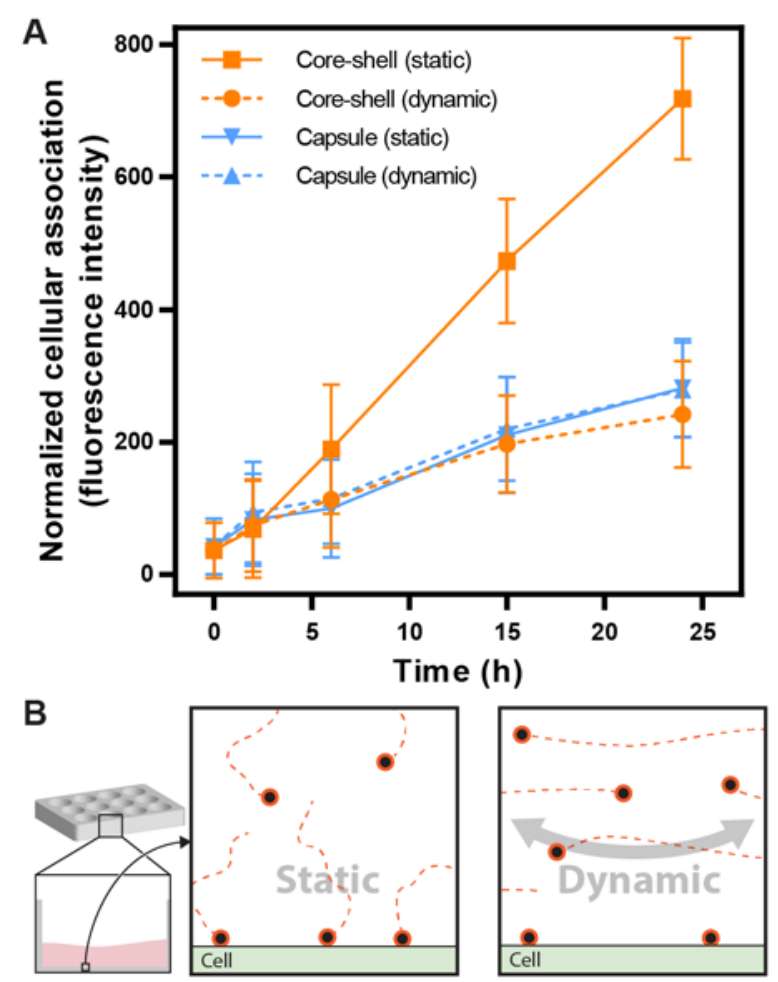

Figure 4. Dynamic flow conditions reduce sedimentation-based effects of cell-particle association. A) Cell-particle association, as assessed by using flow cytometry after incubation of NIH/3T3 cells with fluorescently labeled core-shell particles or capsules under either static or dynamic flow conditions (particle diameter $\sim 0.2 \mu \mathrm{m}$, Table 1). Data points represent the median and error bars indicate the median absolute deviation of the fluorescent cell population. B) Scheme illustrating possible explanation for observed reduction in cell-particle association where dynamic flow conditions introduce mixing that substantially reduces sedimentation effects.

Inspired by reports of how particle shape can influence cellular association, especially under flow, ${ }^{29,30}$ we investigated how soft polymer capsules with different aspect ratios (ARs) interact with cells under dynamic flow conditions. Three types of PMASH capsules with different ARs (spheres, $670 \mathrm{~nm}$ diameter, $\mathrm{AR}=1$; short rods, $310 \mathrm{~nm} \times 2 \mu \mathrm{m}$, AR = 7; long rods, $300 \mathrm{~nm}$ 
$\times 7 \mu \mathrm{m}, \mathrm{AR}=24$ ) were engineered using a silica templating method (Figure 5, Table 2, and Table S1). ${ }^{34}$ These capsules were incubated with cells under both standard static conditions and dynamic flow conditions (Figure 6). The strong fluorescent signal and relative size of these capsules led us to select percent cellular association as our metric for particle comparison. However, in general, percent cellular association is semi-quantitative and caution is advised when using it to compare nanomaterials. Under static conditions (solid lines, Figure 6A), particle shape-dependent cellular association is visible, with cellular association being higher for the rods ( $\sim 90-100 \%)$ than for the spheres ( 60\%) after 24 h. The higher degree of association seen for long rod-shaped particles could be due to their larger dimensions (Table 2) and therefore more pronounced sedimentation, as well as a larger surface area interacting with the cells. ${ }^{29}$

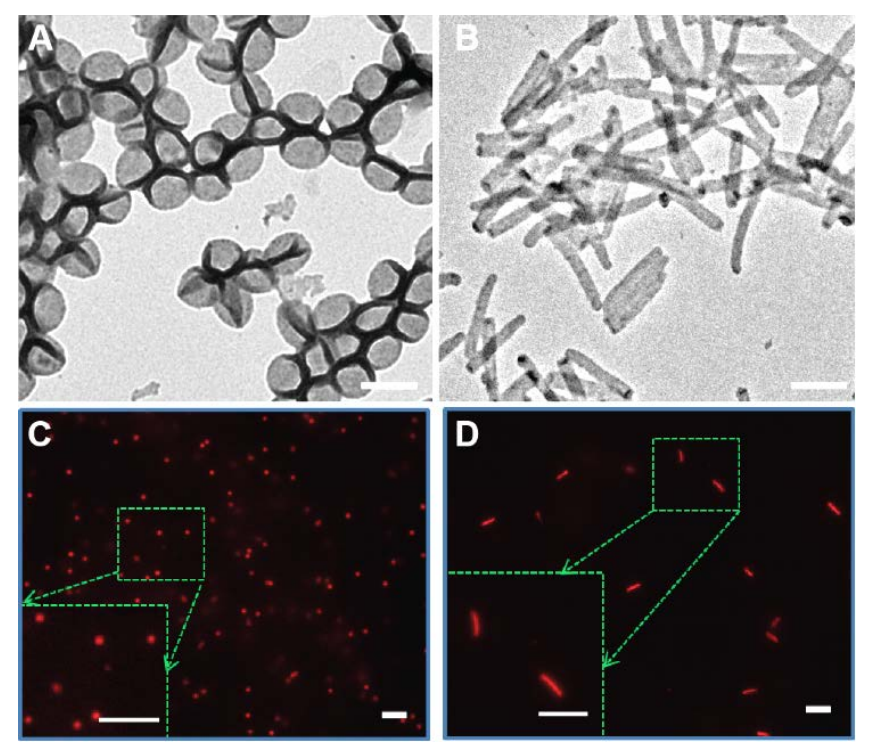

Figure 5. Spherical and rod-shaped $\mathrm{PMA}_{\mathrm{SH}}$ capsules after template dissolution. A,B) TEM images of spherical (A) and rod-shaped (AR = 7) (B) capsules. C,D) Fluorescence microscopy images of fluorescently labeled spherical capsules (C) and rod-shaped (AR = 7) capsules (D) in solution. Insets show higher magnification of indicated regions. See Figure S6 for images of long rod capsules $(A R=24)$. Note that TEM is performed on air-dried capsules in vacuum while 
fluorescence microscopy is performed on hydrated capsules in water. Scale bars are $1.25 \mu \mathrm{m}$ (A, B) and $5 \mu \mathrm{m}(\mathrm{C}, \mathrm{D})$.

Table 2. Dimensions of spherical and rod-shaped PMA $\mathrm{AH}_{\mathrm{SH}}$ capsules

\begin{tabular}{lccc}
\multicolumn{1}{c}{ capsule } & $\begin{array}{c}\text { length } \\
(\mathrm{nm})\end{array}$ & $\begin{array}{c}\text { width } \\
(\mathrm{nm})\end{array}$ & length:width \\
sphere & $670 \pm 60$ & $670 \pm 60$ & $1: 1$ \\
short rod & $2020 \pm 150$ & $310 \pm 30$ & $6.5: 1$ \\
long rod & $7150 \pm 480$ & $300 \pm 40$ & $23.8: 1$
\end{tabular}
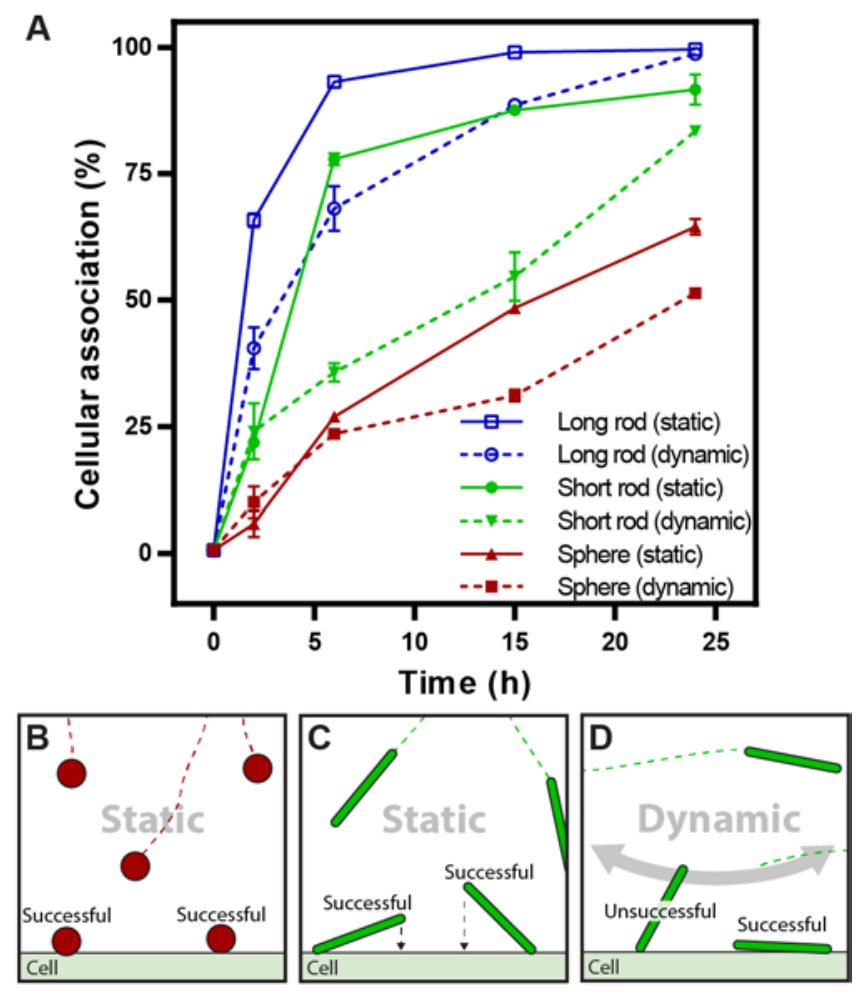

Figure 6. Shape-dependent effects of cell-particle association under dynamic exposure conditions. A) Cell-particle association, as assessed using flow cytometry after incubation of NIH/3T3 cells with either spherical or rod-shaped PMA ${ }_{S H}$ capsules (Table 2) under either static or dynamic flow conditions. Data points represent the percentage of cells positive for capsules 
and error bars indicate the associated standard deviation. B,C,D) Schemes illustrating possible explanation for the observed trends in cell-particle associations.

During dynamic flow conditions, the cellular association for the spheres decreases slightly (from $\sim 60 \%$ to $\sim 50 \%$ ). In addition to reduced sedimentation (as discussed above), a contributing effect could be that the dynamic flow introduces disturbances that re-suspends particles that have loosely associated with the cell surface. For both of the rod-shaped capsules there is a substantial difference between static and dynamic flow conditions at 6 and $15 \mathrm{~h}$. These differences reduce over time and for the long rods both methods result in nearly $100 \%$ of cells having associated with rods after $24 \mathrm{~h}$. For the short rods the largest difference in cellular association between dynamic flow conditions and static conditions is observed after $6 \mathrm{~h}$ ( $78 \%$ for static compared to $35 \%$ for dynamic), and even after $24 \mathrm{~h}$ the cellular association observed for dynamic flow conditions does not fully reach the cellular association observed for static conditions ( $\sim 80 \%$ for dynamic flow conditions compared to $\sim 92 \%$ for static conditions). The differences observed between particle types with different ARs indicate that shape effects are important for cellular association under dynamic flow. While the rod-shaped particles show similar cellular association after $24 \mathrm{~h}$, considerable differences are observed during earlier time points (especially after 6 and 15 h; Figure 6), indicating that not only is the overall shape important (sphere versus rod), but also the AR of the rod shape.

The results observed here are different than those previously observed for filomicelle particles in a microfluidic device. ${ }^{30}$ In that study, ${ }^{30}$ cellular association and uptake decreased for particles with larger AR while herein cellular association increased for particles with larger AR. In addition to the two different particle types used (filomicelles versus layer-by-layer assembled polymer particles), these differences may be explained by the different flow-based devices used. 
In the filomicelle study a microfluidic device with laminar flow was used, which caused particles with larger AR to align with the flow so that larger particles could "flow past" the cells, thus decreasing cellular association. ${ }^{30}$ In contrast, the dynamic mixing device used herein produces chaotic mixing, which does not give rise to this type of laminar flow alignment.

The behavior of rod-shaped particles and spheres under dynamic flow is likely influenced by the multiple modes with which rod-shaped particles contact and interact with cells. While spheres are symmetrical and thus contact cell membranes in the same way, rod-shaped particles can be connected to the cell surface "end-first”, "side-first”, or somewhere in between (Figure 6B-D). When a rod-shaped particle is connected to the cell end-first, such that it protrudes from the surface of the cell, it is more vulnerable to disturbance and detachment due to the external flow. There is theoretical evidence that during endocytosis of particles with a high aspect ratio (i.e., rods), particles transition through an end-first orientation. ${ }^{36,37}$ This may explain why rods see a substantial drop in cellular association under dynamic conditions. The soft nature of the polymer capsules and the dynamic cellular interface further complicates these interactions (as it has been shown that mechanical properties can influence cellular association and uptake) ${ }^{38}$ and additional studies are needed to fully understand the processes involved. Confocal laser scanning microscopy was also performed but did not show any substantial differences between the samples (Figure S7); this is not unexpected as these were obtained from cells after they had been removed from the mixing device, fluorescently labeled, and washed. Ideally imaging should be performed in situ during dynamic flow, and we plan to explore this in future designs.

\section{CONCLUSIONS}

In summary, we investigate fundamental cell-particle interactions under dynamic flow conditions using a simple and self-contained device for creating dynamic flow conditions in 
standard multiwell cell culture plates. We used this device to investigate the effects of particle shape and sedimentation on cellular association for two particle systems and we show that dynamic flow can be used to reduce sedimentation-based effects by comparing two almost identical particle types (excluding density), thus providing a new experimental platform to compare particles. Further, we use three polymer capsules with different aspect ratios and show that the geometry can significantly affect cellular association, and that this effect is different under dynamic flow conditions compared to static conditions. Shape effects under flow-based conditions have proven important for targeting tissues in vivo ${ }^{29}$ and finding ways to investigate these in vitro have the potential to provide new insights valuable for the design and development of improved particle systems for biomedical applications. Taken together, these results demonstrate a simple and rapid way of investigating fundamental bio-interactions of engineered particles in vitro, and provide insight into how sedimentation and particle shape effects affect cellular association under dynamic flow conditions.

\section{ASSOCIATED CONTENT}

Supporting Information. Supporting information, supporting figures, and code for microcontroller. The Supporting Information is available free of charge on the ACS Publications website.

\section{AUTHOR INFORMATION}

\section{Corresponding Author}

*E-mail: fcaruso@unimelb.edu.au

\section{Notes}


The authors declare no competing financial interest.

\section{ACKNOWLEDGMENT}

This work was supported by the Australian Research Council (ARC) under the Australian Laureate Fellowship scheme (F.C., FL120100030), and the ARC Centre of Excellence in Convergent Bio-Nano Science and Technology (Project Number CE140100036). This work was performed in part at the Materials Characterisation and Fabrication Platform (MCFP) at the University of Melbourne and the Victorian Node of the Australian National Fabrication Facility (ANFF).

\section{REFERENCES}

(1) Sherwood, L. Human Physiology: From Cells to Systems, 9th ed.; Cengage Learning: Boston, MA, USA, 2015.

(2) Gabe, I. T.; Gault, J. H.; Ross, J.; Mason, D. T.; Mills, C. J.; Schillingford, J. P.; Braunwald, E. Measurement of Instantaneous Blood Flow Velocity and Pressure in Conscious Man with a Catheter-Tip Velocity Probe. Circulation 1969, 40, 603-614.

(3) Fung, Y. C.; Zweifach, B. W. Microcirculation: Mechanics of Blood Flow in Capillaries. Annu. Rev. Fluid Mech. 1971, 3, 189-210.

(4) Chary, S. R.; Jain, R. K. Direct Measurement of Interstitial Convection and Diffusion of Albumin in Normal and Neoplastic Tissues by Fluorescence Photobleaching. Proc. Natl. Acad. Sci. U. S. A. 1989, 86, 5385-5389.

(5) Swartz, M. A.; Fleury, M. E. Interstitial Flow and Its Effects in Soft Tissues. Annu. Rev. Biomed. Eng. 2007, 9, 229-256.

(6) Mitragotri, S.; Anderson, D. G.; Chen, X.; Chow, E. K.; Ho, D.; Kabanov, A. V; Karp, J. M.; Kataoka, K.; Mirkin, C. A.; Petrosko, S. H.; Shi, J.; Stevens, M. M.; Sun, S.; Teoh, S.; 
Venkatraman, S. S.; Xia, Y.; Wang, S.; Gu, Z.; Xu, C. Accelerating the Translation of Nanomaterials in Biomedicine. ACS Nano 2015, 9, 6644-6654.

(7) De Koker, S.; Hoogenboom, R.; De Geest, B. G. Polymeric Multilayer Capsules for Drug Delivery. Chem. Soc. Rev. 2012, 41, 2867-2884.

(8) Elsabahy, M.; Heo, G. S.; Lim, S.-M.; Sun, G.; Wooley, K. L. Polymeric Nanostructures for Imaging and Therapy. Chem. Rev. 2015, 115, 10967-11011.

(9) Cui, J.; Richardson, J. J.; Björnmalm, M.; Faria, M.; Caruso, F. Nanoengineered Templated Polymer Particles: Navigating the Biological Realm. Acc. Chem. Res. 2016, 49, 1139-1148.

(10) Blanco, E.; Shen, H.; Ferrari, M. Principles of Nanoparticle Design for Overcoming Biological Barriers to Drug Delivery. Nat. Biotechnol. 2015, 33, 941-951.

(11) Nel, A. E.; Mädler, L.; Velegol, D.; Xia, T.; Hoek, E. M. V; Somasundaran, P.; Klaessig, F.; Castranova, V.; Thompson, M. Understanding Biophysicochemical Interactions at the Nano-Bio Interface. Nat. Mater. 2009, 8, 543-557.

(12) Yan, Y.; Björnmalm, M.; Caruso, F. Assembly of Layer-by-Layer Particles and Their Interactions with Biological Systems. Chem. Mater. 2014, 26, 452-460.

(13) Albanese, A.; Tang, P. S.; Chan, W. C. W. The Effect of Nanoparticle Size, Shape, and Surface Chemistry on Biological Systems. Annu. Rev. Biomed. Eng. 2012, 14, 1-16.

(14) Petros, R. A.; DeSimone, J. M. Strategies in the Design of Nanoparticles for Therapeutic Applications. Nat. Rev. Drug Discov. 2010, 9, 615-627.

(15) Zhu, M.; Nie, G.; Meng, H.; Xia, T.; Nel, A.; Zhao, Y. Physicochemical Properties Determine Nanomaterial Cellular Uptake, Transport, and Fate. Acc. Chem. Res. 2013, 46, 622-631. 
(16) Björnmalm, M.; Yan, Y.; Caruso, F. Engineering and Evaluating Drug Delivery Particles in Microfluidic Devices. J. Control. Release 2014, 190, 139-149.

(17) Cho, E. C.; Zhang, Q.; Xia, Y. The Effect of Sedimentation and Diffusion on Cellular Uptake of Gold Nanoparticles. Nat. Nanotechnol. 2011, 6, 385-391.

(18) Cui, J.; Faria, M.; Björnmalm, M.; Ju, Y.; Suma, T.; Gunawan, S. T.; Richardson, J. J.; Heidari, H.; Bals, S.; Crampin, E. J.; Caruso, F. A Framework to Account for Sedimentation and Diffusion in Particle-Cell Interactions. Langmuir 2016, doi: 10.1021/acs.langmuir.6b01634.

(19) Cohen, J. M.; DeLoid, G. M.; Demokritou, P. A Critical Review of in Vitro Dosimetry for Engineered Nanomaterials. Nanomedicine 2015, 10, 3015-3032.

(20) Valencia, P. M.; Farokhzad, O. C.; Karnik, R.; Langer, R. Microfluidic Technologies for Accelerating the Clinical Translation of Nanoparticles. Nat. Nanotechnol. 2012, 7, 623629.

(21) Sackmann, E. K.; Fulton, A. L.; Beebe, D. J. The Present and Future Role of Microfluidics in Biomedical Research. Nature 2014, 507, 181-189.

(22) Albanese, A.; Lam, A. K.; Sykes, E. A.; Rocheleau, J. V; Chan, W. C. W. Tumour-on-aChip Provides an Optical Window into Nanoparticle Tissue Transport. Nat. Commun. 2013, 4, 2718.

(23) Raeesi, V.; Chan, W. C. W. Improving Nanoparticle Diffusion through Tumor Collagen Matrix by Photo-Thermal Gold Nanorods. Nanoscale 2016, 8, 12524-12530.

(24) Korin, N.; Kanapathipillai, M.; Matthews, B. D.; Crescente, M.; Brill, A.; Mammoto, T.; Ghosh, K.; Jurek, S.; Bencherif, S. A.; Bhatta, D.; Coskun, A. U.; Feldman, C. L.; Wagner, D. D.; Ingber, D. E. Shear-Activated Nanotherapeutics for Drug Targeting to 
Obstructed Blood Vessels. Science 2012, 337, 738-742.

(25) Haghgooie, R.; Toner, M.; Doyle, P. S. Squishy Non-Spherical Hydrogel Microparticles. Macromol. Rapid Commun. 2010, 31, 128-134.

(26) Sun, H.; Björnmalm, M.; Cui, J.; Wong, E. H. H.; Dai, Y.; Dai, Q.; Qiao, G. G.; Caruso, F. Structure Governs the Deformability of Polymer Particles in a Microfluidic Blood Capillary Model. ACS Macro Lett. 2015, 4, 1205-1209.

(27) Merkel, T. J.; Jones, S. W.; Herlihy, K. P.; Kersey, F. R.; Shields, A. R.; Napier, M.; Luft, J. C.; Wu, H.; Zamboni, W. C.; Wang, A. Z.; Bear, J. E.; DeSimone, J. M. Using Mechanobiological Mimicry of Red Blood Cells to Extend Circulation Times of Hydrogel Microparticles. Proc. Natl. Acad. Sci. U. S. A. 2011, 108, 586-591.

(28) Cui, J.; Björnmalm, M.; Liang, K.; Xu, C.; Best, J. P.; Zhang, X.; Caruso, F. Super-Soft Hydrogel Particles with Tunable Elasticity in a Microfluidic Blood Capillary Model. Adv. Mater. 2014, 26, 7295-7299.

(29) Kolhar, P.; Anselmo, A. C.; Gupta, V.; Pant, K.; Prabhakarpandian, B.; Ruoslahti, E.; Mitragotri, S. Using Shape Effects to Target Antibody-Coated Nanoparticles to Lung and Brain Endothelium. Proc. Natl. Acad. Sci. U. S. A. 2013, 110, 10753-10758.

(30) Geng, Y.; Dalhaimer, P.; Cai, S.; Tsai, R.; Tewari, M.; Minko, T.; Discher, D. E. Shape Effects of Filaments versus Spherical Particles in Flow and Drug Delivery. Nat. Nanotechnol. 2007, 2, 249-255.

(31) Trebbin, M.; Steinhauser, D.; Perlich, J.; Buffet, A.; Roth, S. V; Zimmermann, W.; Thiele, J.; Förster, S. Anisotropic Particles Align Perpendicular to the Flow Direction in Narrow Microchannels. Proc. Natl. Acad. Sci. U. S. A. 2013, 110, 6706-6711.

(32) Squires, T. M.; Quake, S. R. Microfluidics: Fluid Physics at the Nanoliter Scale. Rev. 
Mod. Phys. 2005, 77, 977-1026.

(33) Lee, C.-Y.; Chang, C.-L.; Wang, Y.-N.; Fu, L.-M. Microfluidic Mixing: A Review. Int. J. Mol. Sci. 2011, 12, 3263-3287.

(34) Chen, X.; Yan, Y.; Müllner, M.; Ping, Y.; Cui, J.; Kempe, K.; Cortez-Jugo, C.; Caruso, F. Shape-Dependent Activation of Cytokine Secretion by Polymer Capsules in Human Monocyte-Derived Macrophages. Biomacromolecules 2016, 17, 1205-1212.

(35) Richardson, J. J.; Björnmalm, M.; Caruso, F. Technology-Driven Layer-by-Layer Assembly of Nanofilms. Science 2015, 348, aaa2491.

(36) Huang, C.; Zhang, Y.; Yuan, H.; Gao, H.; Zhang, S. Role of Nanoparticle Geometry in Endocytosis: Laying Down to Stand Up. Nano Lett. 2013, 13, 4546-4550.

(37) Zhang, S.; Gao, H.; Bao, G. Physical Principles of Nanoparticle Cellular Endocytosis. ACS Nano 2015, 9, 8655-8671.

(38) Banquy, X.; Suarez, F.; Argaw, A.; Rabanel, J.-M.; Grutter, P.; Bouchard, J.-F.; Hildgen, P.; Giasson, S. Effect of Mechanical Properties of Hydrogel Nanoparticles on Macrophage Cell Uptake. Soft Matter 2009, 5, 3984-3991. 
Table of Contents Graphic

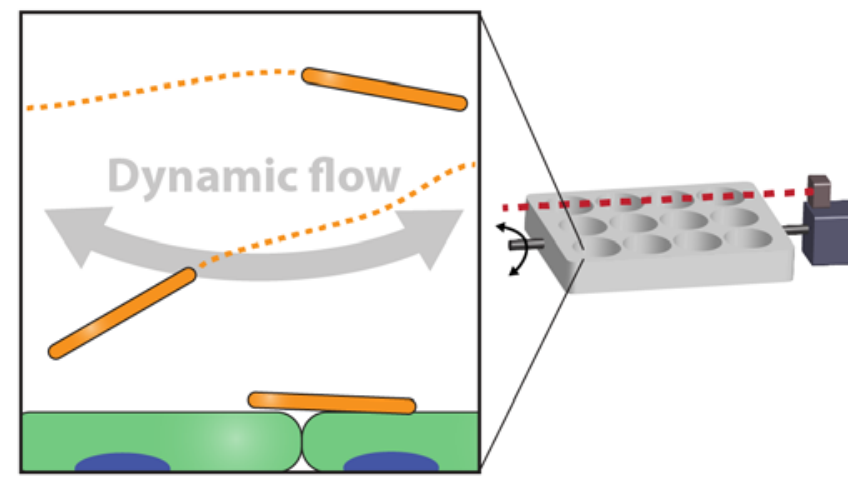




\section{University Library}

\section{- M M N E R VA A gateway to Melbourne's research publications}

Minerva Access is the Institutional Repository of The University of Melbourne

Author/s:

Bjornmalm, M;Faria, M;Chen, X;Cui, J;Caruso, F

Title:

Dynamic Flow Impacts Cell-Particle Interactions: Sedimentation and Particle Shape Effects

Date:

2016-10-25

Citation:

Bjornmalm, M., Faria, M., Chen, X., Cui, J. \& Caruso, F. (2016). Dynamic Flow Impacts

Cell-Particle Interactions: Sedimentation and Particle Shape Effects. LANGMUIR, 32 (42),

pp.10995-11001. https://doi.org/10.1021/acs.langmuir.6b03216.

Persistent Link:

http://hdl.handle.net/11343/120180 